\title{
Exact M-Theory Solutions, Integrable Systems, and Superalgebras ${ }^{\star}$
}

\author{
Eric D'HOKER
}

Department of Physics and Astronomy, University of California, Los Angeles, CA 90095, USA

E-mail: dhoker@physics.ucla.edu

URL: http://www.pa.ucla.edu/directory/eric-dhoker

Received January 05, 2015, in final form April 03, 2015; Published online April 11, 2015

http://dx.doi.org/10.3842/SIGMA.2015.029

\begin{abstract}
In this paper, an overview is presented of the recent construction of fully backreacted half-BPS solutions in 11-dimensional supergravity which correspond to near-horizon geometries of M2 branes ending on, or intersecting with, M5 and M5' branes along a self-dual string. These solutions have space-time manifold $\mathrm{AdS}_{3} \times S^{3} \times S^{3}$ warped over a Riemann surface $\Sigma$, and are invariant under the exceptional Lie superalgebra $D(2,1 ; \gamma) \oplus D(2,1 ; \gamma)$, where $\gamma$ is a real continuous parameter and $|\gamma|$ is governed by the ratio of the number of M5 and M5' branes. The construction proceeds by mapping the reduced BPS equations onto an integrable field theory on $\Sigma$ which is of the Liouville sine-Gordon type. Families of regular solutions are distinguished by the sign of $\gamma$, and include a two-parameter Janus solution for $\gamma>0$, and self-dual strings on M5 as well as asymptotically $\mathrm{AdS}_{4} / \mathbb{Z}_{2}$ solutions for $\gamma<0$.
\end{abstract}

Key words: M-theory; branes; supersymmetry; superalgebras; integrable systems

2010 Mathematics Subject Classification: 81Q60; 17B80

\section{Introduction}

The main theme of my collaboration with Luc Vinet in the mid 1980s was the study of dynamical supersymmetries and associated Lie superalgebras in certain integrable quantum mechanical systems involving magnetic monopoles and dyons. In our first joint paper [23], we showed that the standard Pauli equation for a non-relativistic spinor in the presence of a background Dirac magnetic monopole exhibits a dynamical supersymmetry. The corresponding supercharges close onto the conformal symmetry of the Dirac magnetic monopole, thereby producing the Lie superalgebra $\operatorname{OSp}(1 \mid 2)$. In a series of subsequent papers [24, 25], we extended these results to integrable systems which include a dyon as well as $1 / r^{2}$ and $1 / r$ potentials, established the presence of associated dynamical supersymmetries and higher rank Lie superalgebras, and we solved the spectra using purely group theoretic methods.

The main theme of the present paper is related to the subject of my earlier work with Luc Vinet, in the sense that it deals with integrable systems, dynamical supersymmetries, and Lie superalgebras, albeit now in the context of 11-dimensional supergravity instead of mechanical systems with a finite number of degrees of freedom. Specifically, we shall present an overview of recent work in which exact solutions with $\mathrm{SO}(2,2) \times \mathrm{SO}(4) \times \mathrm{SO}(4)$ isometry and 16 residual supersymmetries (so-called half-BPS solutions) to 11-dimensional supergravity are constructed on space-time manifolds of the form

$$
\left(\mathrm{AdS}_{3} \times S^{3} \times S^{3}\right) \ltimes \Sigma .
$$

${ }^{\star}$ This paper is a contribution to the Special Issue on Exact Solvability and Symmetry Avatars in honour of Luc Vinet. The full collection is available at http://www.emis.de/journals/SIGMA/ESSA2014.html 
The radii of the anti-de-Sitter space $\mathrm{AdS}_{3}$ and of the spheres $S^{3}$ are functions of the twodimensional Riemann surface $\Sigma$, so that the product $\ltimes$ with $\Sigma$ is warped. The construction of [16] proceeds by reducing the BPS equations of 11-dimensional supergravity to the spacetime (1.1), and then mapping the reduced BPS equations onto an integrable 2-dimensional field theory which is a close cousin of the Liouville and sine-Gordon theories. Although large families of exact solutions to this field theory, and correspondingly to the supergravity problem, have been constructed in $[17,18,27]$, a full understanding and analysis of its integrability properties remains to be achieved.

The motivation for this work derives from M-theory, string theory, and gauge/gravity duality as realized by holography. For introductions to these topics and their interrelation, we refer the reader to $[3,20,26,29]$. The basic constituents of M-theory are the M2 and M5 branes with world volumes of respective dimensions $2+1$ and $5+1$. The metric and other fields for one M2 brane, or for a stack of parallel M2 branes, is known analytically in the supergravity approximation. The same holds for M5 branes. But an analytical solution for the intersection of M2 and M5 branes continues to elude us, although solutions with smeared branes have been obtained, and their form is surprisingly simple [34, 44, 48] (see also [35]).

In the work reviewed here, it is shown that half-BPS solutions of M2 branes which end on M5 branes or intersect with them, may be constructed analytically and explicitly, in the near-horizon limit.

In this presentation to an audience of specialists in integrable systems and Lie algebras and superalgebras, however, we shall emphasize integrability and group theory, and further expand upon those topics. For the physical significance of the solutions, within the context of brane intersections and holography, we refer the reader to the recent paper [6], where these properties are addressed in full.

\section{M-theory synopsis}

M-theory unifies the five critical superstring theories, namely Type I, IIA, IIB, Heterotic $E_{8} \times E_{8}$, and Heterotic $\mathrm{SO}(32)$, and provides a natural geometric framework for the unification of the dualities between those superstring theories and some of their compactfications [46]. As Mtheory contains gravity, its basic length scale $\ell$ is set by Newton's constant. Since M-theory provides a complete unification, it has no dimensionless free couplings.

M-theory permits a perturbative treatment in a space-time which itself has a finite typical length scale, which we shall designate by $R$. (Note that this condition is not fulfilled by 11-dimensional flat Minkowski space-time.) The perturbation expansion corresponds to the limit where $R$ is either small or large compared to $\ell$. It is in those limits that M-theory is best-understood. Two classic cases are as follows. First, when 11-dimensional space-time is compactified on a circle of radius $R$, then M-theory coincides with Type IIA superstring theory with string coupling $R / \ell$, and admits a perturbative expansion for $R / \ell \ll 1$. Second, when the fluctuations of the metric and other fields are of a typical length $R$ which is large compared to $\ell$, M-theory admits a perturbative expansion in powers of $\ell / R$ around 11-dimensional supergravity.

The Type IIA approximation to M-theory gives access to the dynamics of the theory at all energy scales, including very high energy at the Planck scale, and is therefore of great conceptual value. Most questions of physical importance, including compactification to 4 space-time dimensions, however, involve much lower energy scales, for which the supergravity limit may be trusted. Moreover, the 11-dimensional supergravity approximation to M-theory and its compactifications faithfully preserves dualities, and provides a calculable framework for gauge/gravity duality via holography. 


\section{$2.1 \quad$ M2 and M5 branes}

The fundamental constituents of M-theory are M2 branes and M5 branes. An Mp brane (for $p=2,5)$ is an extended object in M-theory whose worldvolume has dimension $1+p$, so that $p$ is the spacial dimension of the brane, while the additional 1 accounts for the time dimension on the brane. In 11-dimensional supergravity, whose bosonic field contents consists of a metric $d s^{2}$ and a 4-form field strength $F=d C$ as we shall see more explicitly below, M5 branes carry magnetic charge $N_{5}$ of $F$, while M2 branes carry electric charge, defined by

$$
N_{2}=\frac{1}{4 \pi^{4}} \oint_{\mathcal{C}_{2}}\left(\star F+\frac{1}{2} C \wedge F\right), \quad N_{5}=\frac{1}{2 \pi^{2}} \oint_{\mathcal{C}_{5}} F
$$

for basic homology cycles $\mathcal{C}_{2}$ and $\mathcal{C}_{5}$ of dimensions 7 and 4 respectively. These charges are quantized, so that $N_{2}$ and $N_{5}$ are integers. Therefore, it makes sense to refer to the configuration with $N_{2}=1$ as a single M2 brane and to $N_{2}>1$ as a stack of $N_{2}$ parallel M2 branes. Analogously, $N_{5}=1$ is the single M5 brane, while $N_{5}>1$ corresponds to s stack of $N_{5}$ parallel M5 branes.

Mp branes living in flat Minkowski space-time are represented by fairly simple classical solutions which bear some similarity to the Schwarzschild solution in pure gravity. The Mp brane solutions have the geometry $\mathbb{R}^{1, p} \ltimes \mathbb{R}^{10-p}$, and are invariant under Poincaré transformation on $\mathbb{R}^{1, p}$. Corresponding to this product structure, we shall introduce coordinates $x^{\mu}$ along the brane with $\mu=0,1, \ldots, p$ as well as coordinates orthogonal to the brane which we represent by a $10-p$ dimensional vector $\mathbf{y}$. M2 and M5 branes respectively have the following metrics

$$
\begin{array}{ll}
\text { M2 } & d s^{2}=\left(1+\frac{c_{2} N_{2} \ell^{6}}{y^{6}}\right)^{-\frac{2}{3}} d x^{\mu} d x_{\mu}+\left(1+\frac{c_{2} N_{2} \ell^{6}}{y^{6}}\right)^{+\frac{1}{3}} d \mathbf{y}^{2}, \\
\text { M5 } & d s^{2}=\left(1+\frac{c_{5} N_{5} \ell^{3}}{y^{3}}\right)^{-\frac{1}{3}} d x^{\mu} d x_{\mu}+\left(1+\frac{c_{5} N_{5} \ell^{3}}{y^{3}}\right)^{+\frac{2}{3}} d \mathbf{y}^{2} .
\end{array}
$$

Here, $y=|\mathbf{y}|$ is the flat Euclidean distance to the brane; $d x^{\mu}$ is contracted with the flat Minkowski metric with signature $(-+\cdots+)$ along the brane; and $c_{2}, c_{5}$ stand for constants which are independent of $N_{2}$ and $N_{5}$.

Flat Minkowski space-time is a solution to 11-dimensional supergravity with 32 Poincaré supercharges. The M2 brane, or more generally, a stack of parallel M2 branes, preserves 16 of those 32 Poincaré supersymmetries. The same is true for a stack of parallel M5 branes.

\subsection{Near-horizon geometry}

The M2 and M5 brane solutions in supergravity are regular, despite the apparent singularity of the metric at $y=0$. To see this, we consider the near-horizon approximation $y^{6} \ll c_{2} N_{2} \ell^{6}$ for M2 branes, and $y^{3} \ll c_{5} N_{5} \ell^{3}$ for M5 branes, in which the metrics reduce to the following expressions (after changing coordinates to $z \sim \ell^{3} / y^{2}$ for M2 and $z^{2} \sim \ell^{3} / y$ for M5)

$$
\begin{aligned}
& \text { M2 } \quad d s^{2}=\left(c_{2} N_{2}\right)^{\frac{1}{3}} \ell^{2}\left(\frac{1}{4} \frac{d z^{2}+d x^{\mu} d x_{\mu}}{z^{2}}+\frac{d \mathbf{y}^{2}-d y^{2}}{y^{2}}\right), \\
& \text { M5 } d s^{2}=\left(c_{5} N_{5}\right)^{\frac{2}{3}} \ell^{2}\left(4 \frac{d z^{2}+d x^{\mu} d x_{\mu}}{z^{2}}+\frac{d \mathbf{y}^{2}-d y^{2}}{y^{2}}\right) .
\end{aligned}
$$

We recognize the metric of $\mathrm{AdS}_{4} \times S^{7}$ with radii proportional to $\left(c_{2} N_{2}\right)^{\frac{1}{6}} \ell$ for M2, and the metric of $\mathrm{AdS}_{7} \times S^{4}$ with radii proportional to $\left(c_{5} N_{5}\right)^{\frac{1}{3}} \ell$ for $\mathrm{M} 5$. We remind the reader that these maximally symmetric spaces are the coset spaces $S^{d+1}=\mathrm{SO}(d+2) / \mathrm{SO}(d+1)$ and $\mathrm{AdS}_{d+1}=$ $\mathrm{SO}(d, 2) / \mathrm{SO}(d, 1)$ for Minkowski signature $A d S$. Note that when $N_{2}, N_{5} \gg 1$, the radii of these 
spaces are large and the curvature is small in units of $\ell$, so that the supergravity approximation to M-theory is indeed trustworthy. Finally, the cycles used to define the electric charge of the M2 brane and the magnetic charge of the M5 brane in (2.1) are homeomorphic to the spheres of the near-horizon limits of these branes, namely respectively $\mathcal{C}_{2}=S^{7}$ and $\mathcal{C}_{5}=S^{4}$.

Gauge/gravity duality is the conjectured holographic equivalence of M-theory on the spacetime $\mathrm{AdS}_{4} \times S^{7}$ with a 3 -dimensional conformal quantum field theory with 32 supercharges but without gravity. This theory is known as ABJM theory, and admits a standard Lagrangian formulation $[2,7,39]$. Analogously, a 6-dimensional conformal quantum field theory with 32 supercharges is expected to exist which is holographically dual to M-theory on $\operatorname{AdS}_{7} \times S^{4}$, but the nature of this theory is still unclear, and it is unlikely that it admits a (standard) Lagrangian formulation.

\section{$3 \quad$ Geometry and symmetries of intersecting branes}

A stack of $N_{2}$ parallel M2 branes has 16 residual Poincaré supersymmetries, which in the nearhorizon limit is enhanced to 32 supersymmetries forming the Lie superalgebra $\operatorname{OSp}(8 \mid 4, \mathbb{R})$. Analogously, a stack of $N_{5}$ parallel M5 branes has 16 residual Poincaré supersymmetries, which in the near-horizon limit become enhanced to 32 supersymmetries forming $\operatorname{OSp}\left(8^{*} \mid 4\right)$.

\subsection{Intersecting branes with residual supersymmetry}

When space-time is populated with a generic collection of M2 and M5 branes, the geometry of the branes will be altered by gravitational and other forces of M-theory, and the population will generically preserve no residual supersymmetry. For special angles between the branes, however, some degree of residual supersymmetry may be preserved. We refer the reader to $[8,10,33,43$, $45]$ for helpful overviews and references to earlier work.

The simplest example is, of course, when the branes are parallel, as we had already discussed earlier. Another example is when the branes have certain mutually orthogonal directions, along with other parallel directions. For a collection of M2 and M5 branes, the simplest such example is obtained when the M2 and M5 branes have 2 parallel directions, all others being orthogonal. We may choose a coordinate system in 11-dimensional space-time in which the M2 brane is along the 012 directions, and the M5 brane along the 013456 directions. This configuration is schematically represented in Table 1, where directions parallel to a brane are indicated with the letter $x$, and the 10-th dimension of space is designated by $\downarrow=10$.

Table 1. Half-BPS intersecting M2 and M5 brane configuration.

\begin{tabular}{|c||c|c|c|c|c|c|c|c|c|c|c|}
\hline branes & 0 & 1 & 2 & 3 & 4 & 5 & 6 & 7 & 8 & 9 & $\emptyset$ \\
\hline \hline M2 & $x$ & $x$ & $x$ & & & & & & & & \\
\hline M5 & $x$ & $x$ & & $x$ & $x$ & $x$ & $x$ & & & & \\
\hline
\end{tabular}

It may be shown that the configuration of Table 1, for a collection of arbitrary numbers $N_{2}$ and $N_{5}$ of M2 and M5-branes respectively, preserves 8 residual Poincaré supersymmetries, and is thus half-BPS. Actually, the configuration of Table 1 is not the most general half-BPS configuration of M2 and M5 branes. Indeed, one may add a stack of $N_{5}^{\prime}$ parallel M5 branes in the direction 01789 Ł, which we shall denote by $\mathrm{M}^{\prime}$ as shown in Table 2 below. The full configuration with stacks of $N_{2}, N_{5}, N_{5}^{\prime} \mathrm{M} 2, \mathrm{M} 5$, and M5' branes respectively preserves 8 Poincaré supersymmetries, and is the most general such BPS configuration.

When $N_{2}, N_{5}, N_{5}^{\prime} \gg 1$, one expects a corresponding supergravity solution to exist, but no exact solution has been obtained so far. (Solutions are available when one or both of the stacks 
Table 2. General half-BPS intersecting M2 and M5 brane configuration.

\begin{tabular}{|c||c|c|c|c|c|c|c|c|c|c|c|}
\hline branes & 0 & 1 & 2 & 3 & 4 & 5 & 6 & 7 & 8 & 9 & $\natural$ \\
\hline \hline M2 & $x$ & $x$ & $x$ & & & & & & & & \\
\hline M5 & $x$ & $x$ & & $x$ & $x$ & $x$ & $x$ & & & & \\
\hline M5 $^{\prime}$ & $x$ & $x$ & & & & & & $x$ & $x$ & $x$ & $x$ \\
\hline
\end{tabular}

of branes are "smeared".) Our goal will be to obtain supergravity solutions not for the entire system of M2 and M5 branes, but only for their near-horizon limit.

\subsection{Symmetries of M2 and M5 and their near-horizon geometry}

The bosonic symmetries of the M2 and M5 branes separately, and of their half-BPS intersection may essentially be read off from Table 2 . The supersymmetric completion of these symmetries is further dictated by the requirement of 32 supercharges for M2 and M5 separately, and 16 supercharges for their half-BPS intersection. We shall begin here by discussing the symmetries of the M2 and M5 branes separately, leaving the case of intersections to the subsequent subsection.

A single M2 brane, or a stack of M2 branes, has a Poincaré symmetry algebra $\operatorname{ISO}(2,1)$ along the M2 brane in the 012 directions, and $\mathrm{SO}(8)$ symmetry in the directions 3456789 4 orthogonal to the brane, giving in total $\mathrm{ISO}(2,1) \oplus \mathrm{SO}(8)$. In the near-horizon limit, the bosonic symmetry $\mathrm{SO}(8)$ is unchanged, but the Poincaré symmetry gets enhanced to the conformal symmetry algebra in $2+1$ dimensions. Using the isomorphism $\mathrm{SO}(2,3)=\mathrm{Sp}(4, \mathbb{R})$, the full bosonic symmetry is then $\mathrm{SO}(8) \oplus \mathrm{Sp}(4, \mathbb{R})$. There is only one Lie superalgebra with this maximal bosonic subalgebra and 32 supercharges, namely $\operatorname{OSp}(8 \mid 4, \mathbb{R})$, and it is the full Lie superalgebra symmetry of the near-horizon space-time $\mathrm{AdS}_{4} \times S^{7}$.

Similarly, a single M5 brane or a stack of M5 branes, has a Poincaré symmetry $\operatorname{ISO}(1,5)$ along the directions 013456 of the M5 brane. Using the isomorphism $\mathrm{SO}(5)=\mathrm{Sp}(4)$, the symmetry in the directions $2789 \natural$ is given by $\operatorname{ISO}(1,5) \oplus \mathrm{Sp}(4)$. In the near-horizon limit, this symmetry gets enhanced to $\mathrm{SO}(2,6) \oplus \mathrm{Sp}(4)$, and extends uniquely to the Lie superalgebra $\operatorname{OSp}(2,6 \mid 4)=\operatorname{OSp}\left(8^{*} \mid 4\right)$, which is the symmetry of the near-horizon space-time $\operatorname{AdS}_{7} \times S^{4}$.

\subsection{Symmetries of half-BPS intersecting branes}

The symmetry algebras for the half-BPS intersection of M2 branes with M5 branes, or for the half-BPS intersection of M2 branes with M5' branes, or for the half-BPS intersection of M2, $\mathrm{M} 5$, and $\mathrm{M} 5^{\prime}$ branes are all the same, as may again be derived by inspecting Table 2. It is given by the Poincaré algebra $\operatorname{ISO}(1,1)$ along the branes in the 01 directions, along with a first $\mathrm{SO}(4)$ in the directions 3456 and a second $\mathrm{SO}(4)$ in the directions 789 , giving the total bosonic symmetry $\operatorname{ISO}(1,1) \oplus \mathrm{SO}(4) \oplus \mathrm{SO}(4)$. In the near-horizon limit, this symmetry gets enhanced to $\mathrm{SO}(2,2) \oplus \mathrm{SO}(4) \oplus \mathrm{SO}(4)$.

Which Lie superalgebras have 16 supercharges and $\mathrm{SO}(2,2) \oplus \mathrm{SO}(4) \oplus \mathrm{SO}(4)$ as maximal bosonic subgroup? No simple Lie superalgebra qualifies. We might have anticipated this result by inspecting the part of space-time on which the bosonic algebra acts, which is $\operatorname{AdS}_{3} \times S^{3} \times S^{3}$. This space is isomorphic to the Lie group $B=\mathrm{SO}(2,1) \times \mathrm{SO}(3) \times \mathrm{SO}(3)$ (up to factors of $\mathbb{Z}_{2}$ ) with Lie algebra $\mathcal{B}=\mathrm{SO}(2,1) \oplus \mathrm{SO}(3) \oplus \mathrm{SO}(3)$. The isometry algebra of the Lie group $B$ is given by the commuting left and right actions of $\mathcal{B}$ on $B$, as it would be for any Lie group, thereby giving the isometry algebra $\mathcal{B} \oplus \mathcal{B}$. The Lie superalgebra we are seeking should follow the same pattern, and should therefore be of the form $\mathcal{G} \oplus \mathcal{G}$, with $\mathcal{B}$ the maximal bosonic subalgebra of $\mathcal{G}$. Recasting $\mathcal{B}$ equivalently as $\mathcal{B}=\mathrm{SO}(4) \oplus \mathrm{Sp}(2, \mathbb{R})$, it is manifest that a first candidate for $\mathcal{G}$ is 
$\mathcal{G}=\operatorname{OSp}(4 \mid 2, \mathbb{R})$. However, $\mathcal{B}$ may also be recast as $\mathcal{B}=\operatorname{SO}\left(4^{*}\right) \oplus \operatorname{USp}(2)$, so an alternative candidate for $\mathcal{G}$ is given by $\mathcal{G}=\operatorname{OSp}\left(4^{*} \mid 2\right)$.

The above candidates are special cases of the general $\mathcal{G}$ which consists of the exceptional Lie superalgebra $\mathcal{G}=D(2,1 ; \gamma)$, specifically its real form whose maximal bosonic subalgebra is $\mathrm{SO}(2,1) \oplus \mathrm{SO}(4)$. The parameter $\gamma$ is real and non-zero. In view of the reflection property $D\left(2,1 ; \gamma^{-1}\right)=D(2,1 ; \gamma)$ for this real form, the range of $\gamma$ may be restricted to the interval

$$
\gamma \in[-1,1]
$$

This hypothesis fits nicely with the results of [42], where $D(2,1 ; \gamma)$ arose as one member in the classification of possible 2-dimensional superconformal field theory invariance algebras. For $\gamma=1$, the exceptional Lie superalgebra $D(2,1 ; \gamma)$ reduces to the classical Lie superalgebra $\operatorname{OSp}(4 \mid 2, \mathbb{R})$, while for $\gamma=-1 / 2$ it reduces to $\operatorname{OSp}\left(4^{*} \mid 2\right)$, so that we recover the earlier two candidates. To summarize, the Lie superalgebra which leaves the half-BPS intersection of M2 branes with M5 and M5' branes invariant is given by

$$
D(2,1 ; \gamma) \oplus D(2,1 ; \gamma) \text {. }
$$

From the explicit supergravity solutions, to be discussed next, we will confirm the symmetry under (3.1), and link the parameter $|\gamma|$ to the ratio of the number of M5 and M5' branes.

\section{BPS solutions in 11-dimensional supergravity}

Having developed the geometry and articulated symmetries of intersecting brane configurations, and of their near-horizon limit, in the preceding section, we shall now move onto deriving exact solutions within the context of 11-dimensional supergravity for these brane intersections, in the near-horizon limit.

\subsection{1-dimensional supergravity}

Supergravity in 11-dimensional space-time has 32 supersymmetries and has a single supermultiplet which contains the metric $d s^{2}=g_{m n} d x^{m} d x^{n}$, a Majorana spinor-valued 1-form gravitino $\psi_{m} d x^{m}$ and a real 4-form field strength $F=\frac{1}{24} F_{m n p q} d x^{m} \wedge d x^{n} \wedge d x^{p} \wedge d x^{q}$, with $m, n, p, q=$ $0,1, \ldots, 9, \natural$. The field $F$ derives from a 3 -form potential $C$ by $F=d C$ and thus obeys the Bianchi identity $d F=0$. The field equations are given by [12]

$$
d(\star F)=\frac{1}{2} F \wedge F, \quad R_{m n}=\frac{1}{12} F_{m p q r} F_{n}{ }^{p q r}+\frac{1}{144} g_{m n} F_{p q r s} F^{p q r s}
$$

up to terms which vanish as the gravitino field $\psi_{m}$ vanishes (and which will not be needed in the sequel). Here, $R_{m n}$ is the Ricci tensor, and $\star F$ denotes the Poincaré dual of $F$. The field equations derive from an action which contains the Einstein-Hilbert term, the standard kinetic term for $F$ term, and a Chern-Simons term for $F$. We shall not need the action here.

The supersymmetry transformations acting on the gravitino field are given by [12]

$$
\delta_{\varepsilon} \psi_{m}=D_{m} \varepsilon+\frac{1}{288}\left(\Gamma_{m}{ }^{n p q r}-8 \delta_{m}{ }^{n} \Gamma^{p q r}\right) F_{n p q r} \varepsilon
$$

up to terms which vanish as $\psi_{m}$ vanishes (and which will not be needed in the sequel). Here, $\varepsilon$ is an arbitrary space-time dependent Majorana spinor supersymmetry transformation parameter, $D_{m} \varepsilon$ stands for the standard covariant derivative on spinors, the Dirac matrices are defined by the Clifford algebra relations $\left\{\Gamma_{m}, \Gamma_{n}\right\}=2 I g_{m n}$, and $\Gamma$ matrices with several lower indices are completely anti-symmetrized in those indices. The supersymmetry transformations of the bosonic fields $(g, F)$ are odd in $\psi$ and thus vanish as $\psi$ vanishes, and they will not be needed here. 


\subsection{Supersymmetric solutions}

Classical solutions are usually considered for vanishing Fermi fields, since classically Fermi fields take values in a Grassmann algebra and have odd grading. Thus, we shall set the gravitino field to zero, $\psi_{m}=0$. The field equations of (4.1) now hold exactly.

A classical solution $(g, F)$ is said to be $B P S$ or supersymmetric provided there exist a non-zero supersymmetry transformations $\varepsilon$ which preserve the condition $\psi_{m}=0$, when the bosonic fields in (4.2) are evaluated on the solution in question. Thus, the central equation in the study of supersymmetric solutions is the so-called BPS-equation

$$
\left(D_{m}+\mathcal{F}_{m}\right) \varepsilon=0, \quad \mathcal{F}_{m}=\frac{1}{288}\left(\Gamma_{m}^{n p q r}-8 \delta_{m}^{n} \Gamma^{p q r}\right) F_{n p q r} .
$$

The vector space of solutions $\mathcal{V}_{\varepsilon}=\mathcal{V}_{\varepsilon}(g, F)$ for the spinor $\varepsilon$ depends upon the values taken by the bosonic fields $(g, F)$. For the flat Minkowski solution with $F=0$, the dimension of $\mathcal{V}_{\varepsilon}$ is maximal and equal to 32, corresponding to 32 Poincaré supersymmetries. For the M2 or M5 brane solutions of (2.2) (along with the corresponding expressions for $F$ which we shall provide later), the dimension of $\mathcal{V}_{\varepsilon}$ is 16 . For the near-horizon limits of these branes given in (2.3), the supersymmetry of the corresponding space-times $\mathrm{AdS}_{4} \times S^{7}$ and $\mathrm{AdS}_{7} \times S^{4}$ is enhanced and the dimension of $\mathcal{V}_{\varepsilon}$ is now 32 , and thus equal to the number of fermionic generators of the superalgebras $\operatorname{OSp}(8 \mid 4, \mathbb{R})$ and $\operatorname{OSp}\left(8^{*} \mid 4\right)$ respectively.

We shall be interested in obtaining classical solutions with 16 supersymmetries, namely for which $\operatorname{dim} \mathcal{V}_{\varepsilon}=16$. Such solutions are referred to as half-BPS.

\subsection{Integrability and the BPS equations}

The BPS equations of (4.3) consist of 11 equations each of which is a 32-component Majorana spinor. This system of 352 equations is subject to 1760 integrability conditions, given by

$$
\left(\frac{1}{4} R_{m n p q} \Gamma^{p q}+D_{m} \mathcal{F}_{n}-D_{n} \mathcal{F}_{m}+\left[\mathcal{F}_{m}, \mathcal{F}_{n}\right]\right) \varepsilon=0
$$

where we have used the fact that the commutator of the spin covariant derivates $D_{m}$ is given by the Riemann tensor, $\left[D_{m}, D_{n}\right] \varepsilon=\frac{1}{4} R_{m n p q} \Gamma^{p q}$. Note that, as equations in $\varepsilon$, the integrability conditions (4.4) are purely algebraic. For generic values of the fields $g$ and $F$, there will be no solutions to (4.4), since a generic field configuration is not supersymmetric.

One may investigate the classification of supergravity configurations $(g, F)$ which satisfy the integrability conditions in (4.4) for a given number of supersymmetries $\operatorname{dim} \mathcal{V}_{\varepsilon}$. This line of attack has proven fruitful, and has given rise to a number of important theorems. It is by now well established that requiring maximal supersymmetry, namely $\operatorname{dim} \mathcal{V}_{\varepsilon}=32$, leads to a small family of solutions, including flat Minkowski space-time, the $A d S \times S$ solutions, and pp-waves [28]. Powerful techniques to analyze the BPS system have been developed in [36] based on the exterior differential algebra of forms constructed out of Killing spinors, and in [40] based on the structure of the holonomy group of (4.3). Increasingly stronger results are being obtained, for example in [38], where it was shown that any solution with $\operatorname{dim} \mathcal{V}_{\varepsilon} \geq 30$ actually has the maximal number of 32 supersymmetries.

An alternative question is for which values of $\operatorname{dim} \mathcal{V}_{\varepsilon}$ the BPS integrability conditions guarantee that a configuration $(g, F)$ satisfies the Bianchi identity $d F=0$ and the field equations (4.1). Given the results of the preceding paragraph, the answer is affirmative for $\operatorname{dim} \mathcal{V}_{\varepsilon} \geq 31$. For the families of solutions with space-time of the form $\operatorname{AdS}_{3} \times S^{3} \times S^{3} \times \Sigma$ and $\operatorname{dim} \mathcal{V}_{\varepsilon}=16$ considered in the present paper, the answer is also affirmative. Similar results hold in Type IIB solutions with 16 supersymmetries $[13,14,15]$. As far as we know, however, the question is open for general families of solutions with $\operatorname{dim} \mathcal{V}_{\varepsilon}=16$. Finally, for $\operatorname{dim} \mathcal{V}_{\varepsilon}<16$, the BPS equations do 
not generally imply all the Bianchi identities and field equations, as explicit counter examples are known.

Viewed in terms of integrability conditions which reproduce all the Bianchi and field equations for $(g, F)$, the BPS system bears some striking similarities with the Lax systems, or flatness conditions, in low-dimensional classical integrable systems. The main difference here is that the dimension is high, namely 11 . The most interesting cases of similarity are when $\operatorname{dim} \mathcal{V}_{\varepsilon}$ is large enough for the BPS equations to imply the Bianchi and field equations, but small enough to allow for large families of solutions. It appears that the case $\operatorname{dim} \mathcal{V}_{\varepsilon}=16$ satisfies both requirements, as we shall show next.

\section{Solving the Half-BPS equations}

In this section, we shall show that the BPS equations for the geometry of the half-BPS intersecting branes in the near-horizon limit may be mapped onto a classical integrable conformal field theory in 2 dimensions of the Liouville sine-Gordon type.

\subsection{The Ansatz for space-time and fields}

The bosonic symmetry algebra $\mathrm{SO}(2,2) \oplus \mathrm{SO}(4) \oplus \mathrm{SO}(4)$ of the half-BPS intersecting brane configuration in the near-horizon limit dictates the structure of the space-time manifold of the solution to be of the form

$$
\left(\mathrm{AdS}_{3} \times S_{2}^{3} \times S_{3}^{3}\right) \ltimes \Sigma .
$$

Here, $S_{2}^{3}$ and $S_{3}^{3}$ are two different 3 -spheres, $\Sigma$ is a Riemann surface with boundary, and the product $\ltimes$ is warped in the sense that the radii of the spaces $\mathrm{AdS}_{3}, S_{2}^{3}$, and $S_{3}^{3}$ are all functions of $\Sigma$. The action of the isometry algebra $\mathrm{SO}(2,2) \oplus \mathrm{SO}(4) \oplus \mathrm{SO}(4)$ is on the space $\mathrm{AdS}_{3} \times S_{2}^{3} \times S_{3}^{3}$, for every point on $\Sigma$.

The bosonic fields invariant under $\mathrm{SO}(2,2) \oplus \mathrm{SO}(4) \oplus \mathrm{SO}(4)$ may be parametrized by

$$
\begin{aligned}
& d s^{2}=f_{1}^{2} d s_{\mathrm{AdS}_{3}}^{2}+f_{2}^{2} d s_{S_{2}^{3}}^{2}+f_{3}^{2} d s_{S_{3}^{3}}^{2}+d s_{\Sigma}^{2}, \\
& F=d b_{1} \wedge \omega_{\mathrm{AdS}_{3}}+d b_{2} \wedge \omega_{S_{2}^{3}}+d b_{3} \wedge \omega_{S_{3}^{3}}, \\
& C=b_{1} \omega_{\mathrm{AdS}_{3}}+b_{2} \omega_{S_{2}^{3}}+b_{3} \omega_{S_{3}^{3}} .
\end{aligned}
$$

Here, $d s_{\mathrm{AdS}_{3}}^{2}$ is the $\mathrm{SO}(2,2)$-invariant metric on $\mathrm{AdS}_{3}$ with radius 1 and $\omega_{\mathrm{AdS}_{3}}$ is its volume form. Similarly, $d s_{S_{a}^{3}}^{2}$ for $a=2,3$ is the $\mathrm{SO}(4)$-invariant metric on $S_{a}^{3}$ with radius 1 and $\omega_{S_{a}^{3}}$ is its volume form. The functions $f_{1}, f_{2}, f_{3}, b_{1}, b_{2}, b_{3}$ are real-valued functions of $\Sigma$, and do not depend on $\mathrm{AdS}_{3} \times S_{2}^{3} \times S_{3}^{3}$. Finally, $d s_{\Sigma}^{2}$ is a Riemannian metric on $\Sigma$.

Since the volume forms $\omega_{\mathrm{AdS}_{3}}$ and $\omega_{S_{a}^{3}}$ are closed, the form $F$ indeed obeys $F=d C$ which in turn automatically satisfies the Bianchi identity.

\subsection{Reduced equations}

The BPS equations of (4.3) may be restricted to bosonic fields of the form given by the $\mathrm{SO}(2,2) \oplus$ $\mathrm{SO}(4) \oplus \mathrm{SO}(4)$-invariant Ansatz of (5.2). The resulting reduced BPS equations are quite involved, but may be reduced to a dependence on the following data:

- a real-valued function $h$ on $\Sigma$;

- a complex-valued function $G$ on $\Sigma$;

- three real constants $c_{1}, c_{2}, c_{3}$ which satisfy $c_{1}+c_{2}+c_{3}=0$. 
The functions $f_{1}, f_{2}, f_{3}, b_{1}, b_{2}, b_{3}$ and the metric $d s_{\Sigma}^{2}$ which parametrize the Ansatz may be expressed in terms of these data with the help of the following composite quantities

$$
\gamma=\frac{c_{2}}{c_{3}}, \quad W_{ \pm}=|G \pm i|^{2}+\gamma^{ \pm 1}(G \bar{G}-1) .
$$

A lengthy calculation then gives the following expressions for the metric factors

$$
\begin{aligned}
f_{2}^{6}=\frac{h^{2} W_{-}(G \bar{G}-1)}{c_{2}^{3} c_{3}^{3} W_{+}^{2}}, & f_{1}^{6}=\frac{h^{2} W_{+} W_{-}}{c_{1}^{6}(G \bar{G}-1)^{2}}, \\
f_{3}^{6}=\frac{h^{2} W_{+}(G \bar{G}-1)}{c_{2}^{3} c_{3}^{3} W_{-}^{2}}, & d s_{\Sigma}^{2}=\frac{|\partial h|^{6} W_{+} W_{-}(G \bar{G}-1)}{c_{2}^{3} c_{3}^{3} h^{4}} .
\end{aligned}
$$

The product of the metric factors is particularly simple, and given by

$$
c_{1} c_{2} c_{3} f_{1} f_{2} f_{3}=\sigma h,
$$

where $\sigma$ may take the values \pm 1 , and will be further specified later. The expressions for the functions $b_{1}, b_{2}, b_{3}$ will be exhibited in equation (5.10) below.

\subsection{Regularity conditions}

The requirements of regularity consist of two parts. First, we have the condition of reality, positivity, and the absence of singularities for the metric factors $f_{1}^{2}, f_{2}^{2}$, and $f_{3}^{2}$ in the interior of $\Sigma$. Second, we have regularity conditions on the boundary of $\Sigma$. Clearly, these conditions require $c_{1}, c_{2}, c_{3}$ and $h$ to be real, as we had already stated earlier, and as we shall continue to assume in the sequel.

The requirements of regularity in the interior of $\Sigma$ are as follows. We must have

1) positivity of $f_{1}^{6}$ which requires $W_{+} W_{-} \geq 0$;

2) positivity of $d s_{\Sigma}^{2}$ which requires $\gamma(G \bar{G}-1) W_{+} W_{-} \geq 0$;

3) positivity of $f_{2}^{6}$ and $f_{3}^{6}$ which requires $\gamma(G \bar{G}-1) W_{ \pm} \geq 0$.

A necessary and sufficient condition for all three requirements above to hold true is

$$
\gamma(G \bar{G}-1) \geq 0
$$

as may be readily verified by inspecting (5.3).

The requirements of regularity at the boundary $\partial \Sigma$ of $\Sigma$ are more delicate. We begin by stressing that $\partial \Sigma$ does not correspond to a boundary of the space-time manifold of the supergravity solution; rather it corresponds to interior points. What is special about the points on $\partial \Sigma$ is that either one or the other three spheres, $S_{2}^{3}$ or $S_{3}^{3}$ shrinks to zero radius there. Such points naturally appear when fibering any unit sphere $S^{n+1}$ over its equal latitude angle $\theta$ in the interval $[0, \pi]$. At each value of $\theta$, we have a sphere $S^{n}$ whose radius varies with $\theta$ and goes to 0 for $\theta=0, \pi$. This behavior is manifest from the relation between the unit radius metrics $d s_{S^{n}}^{2}$ and $d s_{S^{n+1}}^{2}$ in this fibration

$$
d s_{S^{n+1}}^{2}=d \theta^{2}+(\sin \theta)^{2} d s_{S^{n}}^{2} .
$$

From the point of view of the total space $S^{n+1}$ the points $\theta=0, \pi$ are unremarkable.

In the geometry at hand, the boundary $\partial \Sigma$ is 1-dimensional, and the fibration will enter for $S^{4}, S^{7}$, and $\mathrm{AdS}_{7}$ (the fibration of $\mathrm{AdS}_{4}$ over $\mathrm{AdS}_{3}$ has no vanishing points). In each case, $\partial \Sigma$ corresponds to the vanishing of either $f_{2}$ or $f_{3}$, but never of $f_{2}$ and $f_{3}$ simultaneously. Conversely, all points where either $f_{2}$ or $f_{3}$ vanishes belong to $\partial \Sigma$. Applying these considerations to the formulas for the metric factors in (5.3) and (5.4), we derive the following necessary and sufficient regularity conditions at the boundary $\partial \Sigma$ : 
1) $h=0$ on $\partial \Sigma$ in view of $f_{2} f_{3}=0$ there and equation (5.4);

2) $W_{+}=0$ when $f_{3}=0$ and $f_{2} \neq 0$ from the vanishing of $h$ on $\partial \Sigma$;

3) $W_{-}=0$ when $f_{2}=0$ and $f_{3} \neq 0$ from the vanishing of $h$ on $\partial \Sigma$.

It follows from this that if $h=0$ everywhere on the boundary $\partial \Sigma$, and if we assume the supergravity solution, and thus $\Sigma$ to be connected, then the sign of $h$ must be constant throughout $\Sigma$. Without loss of generality, we choose

$$
h>0 \quad \text { in the interior of } \Sigma \text {. }
$$

Finally, we note that the conditions $W_{ \pm}=0$ of points 2) and 3) above are readily solved under the assumption (5.5) with the following result

$$
W_{ \pm}=0 \quad \Leftrightarrow \quad G=\mp i .
$$

\subsection{Differential equations}

The BPS equations (4.3) for bosonic fields given by the Ansatz of (5.2), are solved in part by the equations given in (5.3) and (5.10), provided the functions $h$ and $G$ satisfy the following differential equations

$$
\partial_{w} \partial_{\bar{w}} h=0, \quad h \partial_{w} G=\frac{1}{2}(G+\bar{G}) \partial_{w} h
$$

along with the complex conjugate of the second equation. Here, $w, \bar{w}$ are local complex coordinates, and the above equations are invariant under conformal reparametrizations of $w$.

The second equation in (5.8) guarantees the existence (at least locally) of a real function $\Phi$, defined by the differential equation

$$
\partial_{w} \Phi=\bar{G}\left(\partial_{w} \ln h\right) .
$$

The integrability condition between this equation and its complex conjugate is satisfied as soon as the equations of (5.8) are. In turn, (5.9) and its complex conjugate may be used to eliminate $G$ and $\bar{G}$, which gives the following second order differential equation for $\Phi$

$$
2 \partial_{\bar{w}} \partial_{w} \Phi+\partial_{\bar{w}} \Phi\left(\partial_{w} \ln h\right)-\partial_{w} \Phi\left(\partial_{\bar{w}} \ln h\right)=0 .
$$

It must be remembered, of course, that $G$ must satisfy the inequality (5.5) which in terms of $\Phi$ translates to a rather unusual looking inequality, namely $\gamma\left(\left|\partial_{w} \Phi\right|^{2}-\left|\partial_{w} \ln h\right|^{2}\right)>0$.

\subsection{Flux field solutions}

The components of the potential $C$, namely $b_{1}, b_{2}, b_{3}$ are found to be given as follows

$$
\begin{aligned}
& b_{1}=b_{1}^{0}+\frac{\nu_{1}}{c_{1}^{3}}\left(\frac{h(G+\bar{G})}{G \bar{G}-1}+\left(2+\gamma+\gamma^{-1}-\left(\gamma-\gamma^{-1}\right)\right) \tilde{h}\right), \\
& b_{2}=b_{2}^{0}-\frac{\nu_{2}}{c_{2}^{2} c_{3}}\left(\frac{h(G+\bar{G})}{W_{+}}-\Phi+\tilde{h}\right), \quad b_{3}=b_{3}^{0}+\frac{\nu_{3}}{c_{2} c_{3}^{2}}\left(\frac{h(G+\bar{G})}{W_{-}}-\Phi+\tilde{h}\right) .
\end{aligned}
$$

The arbitrary constants parameters $b_{1}^{0}, b_{2}^{0}, b_{3}^{0}$ account for the residual gauge transformations on the 3 -form field $C$ which are allowed within the Ansatz. The factors $\nu_{1}, \nu_{2}, \nu_{3}$ may take values \pm 1 , but supersymmetry places a constraint on their product

$$
\sigma=-\nu_{1} \nu_{2} \nu_{3}
$$


where $\sigma$ is the sign encountered in equation (5.4). The real-valued function $\Phi$ has already been defined in (5.9), and is determined in terms of $G$ and $h$ up to an additive constant. The function $\tilde{h}$ is the harmonic function dual to the harmonic function $h$ and satisfies

$$
\partial_{\bar{w}}(h+i \tilde{h})=0
$$

along with its complex conjugate equation.

The electric field strength, suitably augmented to a conserved combination in order to account for the presence of the Chern-Simons interaction, may be decomposed on the reduced geometry $\left(\mathrm{AdS}_{3} \times S^{3} \times S^{3}\right) \ltimes \Sigma$, as follows

$$
\star F+\frac{1}{2} C \wedge F=-d \Omega_{1} \wedge \hat{e}^{345678}+d \Omega_{2} \wedge \hat{e}^{678012}+d \Omega_{3} \wedge \hat{e}^{012345} .
$$

The Bianchi identity $d F=0$ and the field equation for $F$ guarantee that the 7 -form on each side is a closed differential form, whence the notations $d \Omega_{a}$ with $a=1,2,3$, with the understanding that $\Omega_{a}$ may or may not be single-valued. Since only the 6 -cycle conjugate to $d \Omega_{1}$ is compact, we shall focus on its properties, and we find

$$
\Omega_{1}=\frac{\sigma \nu_{1}}{c_{2}^{3} c_{3}^{3}}\left(\Omega_{1}^{0}+\Omega_{1}^{s}+\Lambda-\tilde{h} \Phi\right) .
$$

Here, $\Omega_{1}^{0}$ is constant, the function $\Lambda$ satisfies the differential equation

$$
\partial_{w} \Lambda=i h \partial_{w} \Phi-2 i \Phi \partial_{w} h
$$

and the function $\Omega_{1}^{s}$ is given by

$$
\Omega_{1}^{s}=\sum_{ \pm} \frac{h}{2 W_{ \pm}}\left(\gamma^{ \pm 1} h\left(|G|^{2}-1\right)+(\Phi \pm \tilde{h})(G+\bar{G})\right)
$$

The M2 brane charges of a solution are obtained by integrating (5.11) over compact seven-cycles, which consist of the warped product of $S_{2}^{3} \times S_{3}^{3}$ over a curve in $\Sigma$ that is spanned between one point on $\partial \Sigma$ where $f_{3}=0$ and another point on $\partial \Sigma$ where $f_{3}=0$. These charges give the net numbers of M2 branes ending respectively on M5 and M5' branes.

\section{Map to an integrable system}

The equations obeyed by $h$ and $G$ in the interior of $\Sigma$ may be summarized as follows For $h$ we have

$$
\partial_{w} \partial_{\bar{w}} h=0, \quad h>0,
$$

while for $G$ we have

$$
\partial_{w} G=\frac{1}{2}(G+\bar{G}) \partial_{w} \ln h, \quad \gamma(G \bar{G}-1)>0 .
$$

The conditions on the boundary of $\Sigma$ may be summarized as follows

$$
h=0, \quad G= \pm i .
$$

These equations are solvable in the following sense.

For any given $\Sigma$, one begins by solving for $h$ and obtaining a real harmonic function $h$ that is strictly positive in the interior of $\Sigma$ and vanishes on $\partial \Sigma$. The algorithm for doing so 
is routine, as the differential equation, the positivity condition, and the boundary condition obey a superposition principle under addition with positive coefficients. If $h_{1}$ and $h_{2}$ are real harmonic, positive inside $\Sigma$, and vanishing on the boundary $\partial \Sigma$, then so is $\alpha_{1} h_{1}+\alpha_{2} h_{2}$ for any real positive coefficients $\alpha_{1}, \alpha_{2}$ which are not both zero.

Having solved for $h$, we now assume that $h$ is given by one such solution, and we proceed to considering the equations for $G$ and $\bar{G}$, namely the second differential equation in (6.1) along with its complex conjugate. For fixed $h$, these equations are linear in $G$ provided the superposition is carried out with real coefficients. If $G_{1}$ and $G_{2}$ obey the second differential equation in (6.1), then so does $a_{1} G_{1}+a_{2} G_{2}$ for any real $a_{1}, a_{2}$.

However, the positivity condition $\gamma(G \bar{G}-1)>0$ and the boundary condition $G= \pm i$ will not be maintained by such linear superposition, even if with real coefficients. The key reason is that the first condition is not linear. Thus, the linearity of the differential equations for $G$ is obstructed by the non-linearity of the positivity and boundary conditions, and the full problem is genuinely non-linear.

\subsection{Associated integrable system}

To expose the presence of an integrable system, we parametrize the complex function $G$ by polar coordinates in terms of real functions $\psi>0$ and $\theta$

$$
G=\psi e^{i \theta}
$$

The non-linear constraint then reduces to the linear relation

$$
\gamma(\psi-1)>0 \quad \text { in the interior of } \Sigma
$$

while the boundary conditions are also linear, and given by

$$
\psi=1, \quad \theta= \pm \frac{\pi}{2} .
$$

However, the differential equation for $G$, and its complex conjugate equation, expressed in terms of the variables $\psi$ and $\theta$ are now non-linear, and given by

$$
\partial_{w} \ln \psi+i \partial_{w} \theta=\left(1+e^{-i \theta}\right) \partial_{w} \ln h, \quad \partial_{\bar{w}} \ln \psi-i \partial_{\bar{w}} \theta=\left(1+e^{+i \theta}\right) \partial_{\bar{w}} \ln h .
$$

The integrability condition on the system (6.2), viewed as equations for $\theta$, will involve both $\psi$ and $\theta$, and will be of no interest here. When the system (6.2) is viewed as equations for $\psi$, the integrability condition is a second order equation for the field $\theta$ only, and is given by

$$
2 \partial_{\bar{w}} \partial_{w} \theta+2 \sin \theta\left(\partial_{\bar{w}} \partial_{w} \ln h\right)+e^{+i \theta} \partial_{w} \theta\left(\partial_{\bar{w}} \ln h\right)+e^{-i \theta} \partial_{\bar{w}} \theta\left(\partial_{w} \ln h\right)=0 .
$$

This equation is integrable in the classical sense. To see this, one may either interpret (6.2) as a Bäcklund transformation for the equation (6.3), or one may expose a Lax pair associated with (6.3). That Lax pair does indeed exist, and is given as follows

$$
\begin{array}{ll}
L_{w}=\partial_{w}+A_{w}, & A_{w}=+i \partial_{w} \theta-\left(1+e^{-i \theta}\right) \partial_{w} \ln h, \\
L_{\bar{w}}=\partial_{\bar{w}}+A_{\bar{w}}, & A_{\bar{w}}=-i \partial_{\bar{w}} \theta-\left(1+e^{+i \theta}\right) \partial_{\bar{w}} \ln h .
\end{array}
$$

Flatness of the connection $A_{w}, A_{\bar{w}}$ and of the covariant derivatives $L_{w}$ and $L_{\bar{w}}$ implies equation (6.3). The associated Lax equations

$$
L_{w} \psi=L_{\bar{w}} \psi=0
$$


coincide with the set of equations (6.2) that we started with. In summary, equation (6.3) has an associated Lax pair, and is integrable in the classical sense.

Furthermore, equation (6.3) is invariant under conformal reparametrizations of the local complex coordinates $w$ and $\bar{w}$. Using this invariance, one may choose local complex coordinates $w, \bar{w}$ such that $h=\Im(w)$, so that equation (6.3) becomes

$$
2 \partial_{\bar{w}} \partial_{w} \theta+\frac{2}{(w-\bar{w})^{2}} \sin \theta-\frac{1}{w-\bar{w}} e^{+i \theta} \partial_{w} \theta+\frac{1}{w-\bar{w}} e^{-i \theta} \partial_{\bar{w}} \theta=0 .
$$

This equation now depends upon a single real field $\theta$ and is clearly related to the sine-Gordon and Liouville equations [22], specifically the Liouville equation on the upper half plane with the Poincaré constant negative curvature metric

$$
d s_{\Sigma}^{2}=\frac{|d w|^{2}}{\Im(w)^{2}}
$$

as discussed, for example, in [21].

\section{$7 \quad$ Role of the superalgebra $D(2,1 ; \gamma) \oplus D(2,1 ; \gamma)$}

Earlier in this paper, we have stated the expectation that the supersymmetries of the half-BPS solution of intersecting M2, M5, and M5' branes in the near-horizon limit will generate the Lie superalgebra $D(2,1 ; \gamma) \oplus D(2,1 ; \gamma)$. In this section, we shall review the structure of the Lie superalgebra $D(2,1 ; \gamma)$, list some of its properties, and show that it is indeed realized by the solutions obtained above.

\subsection{The Lie superalgebra $D(2,1 ; \gamma)$}

The complex Lie superalgebra $D(2,1 ; \gamma)$ is the only finite-dimensional simple Lie superalgebra that depends on a continuous parameter, namely the complex parameter $\gamma$. The maximal bosonic subalgebra of $D(2,1 ; \gamma)$ is $\operatorname{SL}(2, \mathbb{C}) \oplus \operatorname{SL}(2, \mathbb{C}) \oplus \operatorname{SL}(2, \mathbb{C})$. The smallest classical Lie superalgebra which contains $D(2,1 ; \gamma)$ for all values of $\gamma$ is $\operatorname{OSp}(9 \mid 8)$. An equivalent way of representing $\gamma$ is by three complex numbers $c_{1}, c_{2}, c_{3}$ modulo an overall complex rescaling, which satisfy $c_{1}+c_{2}+c_{3}=0$ and $\gamma=c_{2} / c_{3}$. The six permutations $\sigma \in \mathfrak{S}_{3}$ of the numbers $c_{1}$, $c_{2}, c_{3}$ induce permutations $\sigma(\gamma)=c_{\sigma(2)} / c_{\sigma(3)}$ under which the complex algebra is invariant

$$
D(2,1 ; \sigma(\gamma))=D(2,1 ; \gamma)
$$

The complex Lie superalgebra $D(2,1 ; \gamma)$ has three inequivalent real forms which are denoted $D(2,1 ; \gamma, p)$ for $p=0,1,2$, and which have $\gamma$ real and maximal bosonic subalgebra $\mathrm{SO}(2,1) \oplus$ $\mathrm{SO}(4-p, p)$. The real form of interest here is $D(2,1 ; \gamma, 0)$; its maximal bosonic subalgebra is isomorphic to $\mathrm{SO}(2,1) \oplus \mathrm{SO}(3) \oplus \mathrm{SO}(3)$. The automorphism group $\mathfrak{S}_{3}$ is reduced to the subgroup $\mathfrak{S}_{2}$ which permutes the two $\mathrm{SO}(3)$ algebras, and acts by $\sigma(\gamma)=\gamma^{-1}$.

The generators of the maximal bosonic subalgebra ${ }^{1} \mathrm{SO}(2,1)_{1} \oplus \mathrm{SO}(3)_{2} \oplus \mathrm{SO}(3)_{3}$ of the real form $D(2,1 ; \gamma, 0)$ will be denoted by $T_{i}^{(a)}$, where the index $a=1,2,3$ refers to the simple components of the algebra and the index $i=1,2,3$ labels the three generators corresponding to component $a$. For example, $T_{i}^{(1)}$ are the three generators of $\mathrm{SO}(2,1)_{1}$. The bosonic structure relations are given as follows

$$
\left[T_{i}^{(a)}, T_{j}^{(b)}\right]=i \delta^{a b} \varepsilon_{i j k} \eta_{(a)}^{k \ell} T_{\ell}^{(a)}
$$

\footnotetext{
${ }^{1}$ The labels on the simple factors are introduced in analogy with the notation of the corresponding factor spaces in (5.1) and (5.2).
} 
where $\eta_{(2)}=\eta_{(3)}=\operatorname{diag}(+++)$ are the invariant metrics of $\mathrm{SO}(3)_{2}$ and $\mathrm{SO}(3)_{3}$ and $\eta_{(1)}=$ $\operatorname{diag}(-,+,+)$ is the invariant metric of $\mathrm{SO}(2,1)_{1}$.

The fermionic generators of $D(2,1 ; \gamma, 0)$ transform under the 2-dimensional irreducible representation of each one of the bosonic simple subalgebras. We shall denote these generators by $F$ with components $F_{\alpha_{1}, \alpha_{2}, \alpha_{3}}$ where $\alpha_{a}$ are 2-dimensional spinor indices. This characterization uniquely determines the commutation relations of $T^{(a)}$ with $F$. The remaining structure relations are given by the anti-commutators of $F$ which take the form

$$
\begin{aligned}
\left\{F_{\alpha_{1}, \alpha_{2}, \alpha_{3}}, F_{\beta_{1}, \beta_{2}, \beta_{3}}\right\}= & c_{1}\left(C \sigma^{i}\right)_{\alpha_{1} \beta_{1}} C_{\alpha_{2} \beta_{2}} C_{\alpha_{3} \beta_{3}} T_{i}^{(1)} \\
& +c_{2} C_{\alpha_{1} \beta_{1}}\left(C \sigma^{i}\right)_{\alpha_{2} \beta_{2}} C_{\alpha_{3} \beta_{3}} T_{i}^{(2)}+c_{3} C_{\alpha_{1} \beta_{1}} C_{\alpha_{2} \beta_{2}}\left(C \sigma^{i}\right)_{\alpha_{3} \beta_{3}} T_{i}^{(3)} .
\end{aligned}
$$

Here, $\sigma^{i}$ are the Pauli matrices, $C=i \sigma^{2}, \gamma=c_{2} / c_{3}$, and $c_{1}+c_{2}+c_{3}=0$. For the complex Lie superalgebra $D(2,1 ; \gamma)$, the parameters $c_{1}, c_{2}, c_{3}$ are complex while for the real forms these parameters are real. For the real form $D(2,1 ; \gamma, 0)$, the automorphism $\gamma \rightarrow \gamma^{-1}$ amounts to interchanging the generators with labels $a=2$ and $a=3$.

\subsection{Invariance of the solutions under $D(2,1 ; \gamma, 0) \oplus D(2,1 ; \gamma, 0)$}

In this subsection, we shall summarize the arguments of Appendix D of [19] in which a proof is given of the invariance under $D(2,1 ; \gamma, 0) \oplus D(2,1 ; \gamma, 0)$ of the half-BPS supergravity solutions. It is manifest that all solutions have 9 Killing vectors $v^{m}$ which correspond to the 9 generators of $\mathrm{SO}(2,1)_{1} \oplus \mathrm{SO}(3)_{2} \oplus \mathrm{SO}(3)_{3}$, and which satisfy

$$
\nabla_{m} v_{n}+\nabla_{n} v_{m}=0 \text {. }
$$

By construction, the half-BPS solutions also have 16 supersymmetries, or Killing spinors $\varepsilon$. Nine Killing vectors and sixteen Killing are precisely the correct numbers of bosonic and fermionic generators needed for $D(2,1 ; \gamma, 0) \oplus D(2,1 ; \gamma, 0)$. To prove that these Killing vectors and Killing spinors together generate the algebra $D(2,1 ; \gamma, 0) \oplus D(2,1 ; \gamma, 0)$, we must ensure that the structure relations of $D(2,1 ; \gamma, 0) \oplus D(2,1 ; \gamma, 0)$ are satisfied. This is manifest for the commutation relations of two bosonic generators, and of one bosonic and one fermionic generator. Thus, it remains to show that the composition of two fermionic generators gives the bosonic generators with the correct parameters $c_{1}, c_{2}, c_{3}$.

To obtain the composition law for two Killing spinors $\varepsilon$ and $\varepsilon^{\prime}$, one proceeds as follows. Let $\varepsilon$ and $\varepsilon^{\prime}$ satisfy the BPS equations (4.3), so that they are Killing spinors. One proves, using the same BPS equations, that one has

$$
\nabla_{m}\left(\bar{\varepsilon} \Gamma_{n} \varepsilon^{\prime}\right)=\frac{1}{3}\left(\bar{\varepsilon} \Gamma^{p q} \varepsilon^{\prime}\right) F_{m n p q} .
$$

By symmetrizing both sides in $m$ and $n$, and using the anti-symmetry of $F$ in these indices, one readily finds that the combination

$$
v_{m}=\bar{\varepsilon} \Gamma_{m} \varepsilon^{\prime}
$$

satisfies the Killing vector equation (7.1).

An overall rescaling of $\varepsilon, \varepsilon^{\prime}$ and $v_{m}$ is immaterial in establishing the structure relations of $D(2,1 ; \gamma, 0) \oplus D(2,1 ; \gamma, 0)$, but the relative normalizations of the various generators are crucial to extract the correct ratio $\gamma$. Proper normalization may be enforced as follows. We begin by introducing an invariant basis of 2-component Killing spinors $\chi_{1}^{\eta_{1}}, \chi_{2}^{\eta_{2}}$, and $\chi_{3}^{\eta_{3}}$ respectively of $\mathrm{SO}(2,1)_{1}, \mathrm{SO}(3)_{2}$, and $\mathrm{SO}(3)_{3}$ for $\eta_{a}= \pm 1$ and $a=1,2,3$. The Killing spinors are normalized as follows

$$
-i \bar{\chi}_{1}^{\eta} \chi_{1}^{\eta^{\prime}}=\left(\chi_{2}^{\eta}\right)^{\dagger} \chi_{2}^{\eta^{\prime}}=\left(\chi_{3}^{\eta}\right)^{\dagger} \chi_{3}^{\eta^{\prime}}=\delta^{\eta \eta^{\prime}}
$$


We decompose the 32-component spinor $\varepsilon$ on the tensor product of these basis Killing spinors

$$
\varepsilon=\chi_{1}^{\eta_{1}} \otimes \chi_{2}^{\eta_{2}} \otimes \chi_{3}^{\eta_{3}} \otimes \zeta_{\eta_{1}, \eta_{2}, \eta_{3}}
$$

where, for each assignment of $\eta_{a}$, the spinor $\zeta$ has 4 components. To evaluate the metric factors and the Killing vectors in terms of $\zeta$, we introduce an adapted basis of Dirac matrices, in frame basis, with indices $\mathfrak{a}_{1}=0,1,2, \mathfrak{a}_{2}=3,4,5, \mathfrak{a}_{3}=6,7,8$, and $\mathfrak{a}=9$, $\curvearrowleft$, given by

$$
\begin{array}{ll}
\Gamma^{\mathfrak{a}_{1}}=\gamma^{\mathfrak{a}_{1}} \otimes I_{2} \otimes I_{2} \otimes \sigma^{1} \otimes \sigma^{3}, & \sigma^{1}=\gamma^{1}=\gamma^{4}=\gamma^{7}=\gamma^{9}, \\
\Gamma^{\mathfrak{a}_{2}}=I_{2} \otimes \gamma^{\mathfrak{a}_{2}} \otimes I_{2} \otimes \sigma^{2} \otimes \sigma^{3}, & \sigma^{2}=-i \gamma^{0}=\gamma^{3}=\gamma^{6}=\gamma^{\natural}, \\
\Gamma^{\mathfrak{a}_{3}}=I_{2} \otimes I_{2} \otimes \gamma^{\mathfrak{a}_{3}} \otimes \sigma^{3} \otimes \sigma^{3}, & \sigma^{3}=\gamma^{2}=\gamma^{5}=\gamma^{8}, \\
\Gamma^{\mathfrak{a}}=I_{2} \otimes I_{2} \otimes I_{2} \otimes I_{2} \otimes \gamma^{\mathfrak{a}} . &
\end{array}
$$

The relations between the Killing spinors and vectors may be expressed in this manner as well. We have $\bar{\varepsilon} \Gamma^{\mathfrak{a}} \varepsilon^{\prime}=0$, as well as, for $\eta= \pm$

$$
\begin{aligned}
& \bar{\varepsilon} \Gamma^{\mathfrak{a}_{1}} \varepsilon^{\prime}=+2 c_{1} f_{1}\left(\bar{\chi}_{1}^{\eta_{1}} \gamma^{\mathfrak{a}_{1}} \chi_{1}^{\eta_{1}}\right), \quad \bar{\varepsilon} \Gamma^{\mathfrak{a}_{2}} \varepsilon^{\prime}=-2 i c_{2} f_{2}\left(\left(\chi_{2}^{\eta_{2}}\right)^{\dagger} \gamma^{\mathfrak{a}_{2}} \chi_{2}^{\eta_{2}}\right), \\
& \bar{\varepsilon} \Gamma^{\mathfrak{a}_{3}} \varepsilon^{\prime}=-2 i c_{3} f_{3}\left(\left(\chi_{3}^{\eta_{3}}\right)^{\dagger} \gamma^{\mathfrak{a}_{3}} \chi_{3}^{\eta_{3}}\right) .
\end{aligned}
$$

The Killing vectors $v_{a}^{\eta_{a}}$ are related to the normalized Killing vectors $\hat{v}^{\mathfrak{a}_{a}}$ and to the normalized Killing spinors as follows

$$
\begin{aligned}
& \left(v_{1}^{\eta}\right)^{\mathfrak{a}_{1}}=f_{1}\left(\hat{v}_{1}^{\eta}\right)^{\mathfrak{a}_{1}}=f_{1} \bar{\chi}_{1}^{\eta_{1}} \gamma^{\mathfrak{a}_{1}} \chi_{1}^{\eta_{1}}, \quad\left(v_{2}^{\eta}\right)^{\mathfrak{a}_{2}}=f_{2}\left(\hat{v}_{2}^{\eta}\right)^{\mathfrak{a}_{2}}=f_{2}\left(\chi_{2}^{\eta_{2}}\right)^{\dagger} \gamma^{\mathfrak{a}_{2}} \chi_{2}^{\eta_{2}}, \\
& \left(v_{3}^{\eta}\right)^{\mathfrak{a}_{3}}=f_{3}\left(\hat{v}_{3}^{\eta}\right)^{\mathfrak{a}_{3}}=f_{3}\left(\chi_{3}^{\eta_{3}}\right)^{\dagger} \gamma^{\mathfrak{a}_{1}} \chi_{3}^{\eta_{3}}
\end{aligned}
$$

so that the physical Killing vectors $v^{A}$ are gives as follows

$$
\bar{\varepsilon} \Gamma^{\mathfrak{a}_{1}} \varepsilon^{\prime}=2 c_{1}\left(v_{1}^{\eta_{1}}\right)^{\mathfrak{a}_{1}}, \quad \bar{\varepsilon} \Gamma^{\mathfrak{a}_{2}} \varepsilon^{\prime}=2 c_{2}\left(v_{2}^{\eta_{2}}\right)^{\mathfrak{a}_{2}}, \quad \bar{\varepsilon} \Gamma^{\mathfrak{a}_{3}} \varepsilon^{\prime}=2 c_{3}\left(v_{3}^{\eta_{3}}\right)^{\mathfrak{a}_{3}} .
$$

The parameters $c_{1}, c_{2}$, and $c_{3}$ now naturally emerge in the structure relations, and are seen to reproduce those of the algebra $D(2,1 ; \gamma, 0)_{+} \oplus D(2,1 ; \gamma, 0)_{-}$for the indices $\eta= \pm$. This concludes the proof of the invariance of the solutions under this Lie superalgebra.

\section{Families and moduli spaces of exact solutions}

In this section, we shall give a brief overview of the solutions that have been derived with the help of the approach developed in the preceding sections of this paper, and refer the reader to Sections 7 and 8 of [6] for details and derivations. We begin with four basic results.

1. All solutions arise as families in the parameter $\gamma \in[-1,+1]$, which governs the dependence of the supergravity fields on the $\gamma$-independent data $(h, G)$.

2. Across the value $\gamma=0$ the number of $M 5$ branes tends to zero, which leads to the decompactification of the directions 3456 in Table 2, which in turn corresponds to sending the radius of the sphere $S_{2}^{3}$ to infinity. (A mirror image decompactification takes place at $\gamma=\infty$, where the number of $\mathrm{M} 5^{\prime}$ branes tends to zero and the radius of $S_{3}^{3}$ diverges.) Across the value $\gamma=-1$ all three components decompactify and the spheres are permuted into one another.

3. Solution for which $\Sigma$ is compact without boundary have positive $\gamma$, constant $h, G$, constant $f_{1}, f_{2}, f_{3}$, with $f_{3}^{2}=\gamma f_{2}^{2}$ and $f_{1}^{2}=\gamma f_{2}^{2} /(1+\gamma)$. In this case, the expression for the metric $d s_{\Sigma}^{2}$ needs to be defined with some extra care as the naive form in (5.3) would vanish for constant $h$. 
4. A regular solution with $\gamma<0$ cannot have more than one asymptotic $A d S$ region, and therefore cannot be dual to an interface conformal field theory.

An important implication of point 3) above is the uniqueness of solutions dual to twodimensional conformal field theories, namely they must be of the form $\operatorname{AdS}_{3} \times S^{3} \times S^{3} \times T^{2}$, where $T^{2}$ is a flat two-torus, and the product is direct. This is the near-horizon geometry of M2 branes suspended between M5 branes, in the limit where the M5 branes have been smeared [10]. The implication of point 3) is that the infrared dynamics on the M2 branes always restores the translation symmetry which would otherwise have been broken by te localized M5 branes. This dynamical behavior should be contrasted with the analogous situation of D3 branes suspended between NS5 branes and D5 branes, where the 3-dimensional field theory on the suspended D3 branes has many strongly-coupled infrared fixed points [30, 31, 32], which are in one-to-one correspondence with a rich set of half-BPS solutions of the Type IIB supergravity equations [1, 4, 5].

Under the regularity conditions spelled out in section 5 , for the case where $\Sigma$ has a boundary and away from the values $\gamma=0,-1$, the solutions of the differential equations (5.9) and the boundary conditions (5.6) and (5.7) produce regular, fully back-reacted supergravity nearhorizon limits of half-BPS intersecting M2, M5, and M5' branes.

\subsection{Global solutions with $\gamma>0$}

The maximally supersymmetric solution $\mathrm{AdS}_{4} \times S^{7}$ corresponds to $\gamma=1$. It admits a deformation by one real continuous parameter $\lambda$ to a Janus solution, discovered in [18]. Mapping $\Sigma$ to the upper half complex plane with the real axis as boundary, and using global complex coordinates $w, \bar{w}$ on $\Sigma$, the functions $(h, G)$ are given as follows

$$
h=i\left(\frac{1}{w}-w\right)+\text { c.c. }, \quad G=i \frac{|w|+|w|^{-1}+\lambda(w-\bar{w})|w|^{-1}}{\bar{w}+\bar{w}^{-1}} .
$$

Here, $\lambda$ can take any real value. The undeformed $\mathrm{AdS}_{4} \times S^{7}$ solution corresponds to $\lambda=0$. The corresponding metric functions $f_{1}, f_{2}, f_{3}, d s_{\Sigma}^{2}$ may be obtained for any $\gamma>0$ from the equations of (5.3), and the flux potentials $b_{1}, b_{2}, b_{3}$ may be obtained from (5.10). The solutions describe a superconformal domain wall of the dual gauge theory on the M2 brane. This twoparameter deformation of $\mathrm{AdS}_{4} \times S^{7}$ was discovered by independent means as a solution of gauged 4-dimensional supergravity in [9], with whom we find precise agreement.

\subsubsection{String and semi-infinite M2 branes}

Besides the strictly regular Janus solutions of the preceding paragraph, there are also solution with a mild singularity which have non-vanishing M5 charge and are analogous to the highly curved NS5 brane and D5 brane regions of $[1,4,5]$. Mapping $\Sigma$ again to the upper half plane with complex coordinates $w, \bar{w}$, the functions $(h, G)$ are given by

$$
h=-i(w-\bar{w}), \quad G= \pm G_{0} \pm \sum_{n=1}^{N+1} \frac{\zeta_{n} \Im(w)}{\left(\bar{w}-x_{n}\right)\left|w-x_{n}\right|} .
$$

Here, $N$ is a positive integer, and $x_{n}$ and $\zeta_{n}$ are arbitrary real moduli of the solutions. The function $G_{0}$ may take the values of either $G_{0}=i$, in which case the conformal boundary of the solution is that of a deformed $\operatorname{AdS}_{7} \times S^{4}$ space-time, or $G_{0}$ may take the value $G_{0}=i w /|w|$, in which case the conformal boundary of the solution is that of a deformed $\mathrm{AdS}_{4} / \mathbb{Z}_{2} \times S^{7}$ spacetime. These solutions describe self-dual strings respectively on the world-volume of M5 branes, or semi-infinite M2 branes. 


\subsection{Global solutions with $\gamma<0$}

The maximally supersymmetric solution $\operatorname{AdS}_{7} \times S^{4}$ has $\gamma=-\frac{1}{2}$, and corresponds to the nearhorizon limit of a stack of parallel M5 branes. Mapping $\Sigma$ again to the upper half plane with complex coordinates $w, \bar{w}$, the functions $(h, G)$ for this solution are given by

$$
h=-i(w-\bar{w}), \quad G=i\left(-1+\frac{w+1}{|w+1|}+\frac{w-1}{\mid w-1}\right) .
$$

The effects of taking $\gamma$ away from the point $\gamma=-\frac{1}{2}$ include a deformation of the metric away from the maximally symmetric metric, to one that has only the reduced symmetry $\mathrm{SO}(2,2) \oplus$ $\mathrm{SO}(4) \oplus \mathrm{SO}(4)$, as well as turning on the flux fields associated with M5' and M2 branes, but without generating either a net magnetic or electric charge.

\subsubsection{Self-dual strings and Young tableaux}

The solution of (8.1) was generalized in [17] by the addition of an arbitrary number $2 N$ of cap singularities in $G$ and, for $\Sigma$ the upper half plane with coordinates $w, \bar{w}$, is given by

$$
i G=1+\sum_{n=1}^{2 N+2}(-)^{n} \frac{w-\xi_{n}}{\left|w-\xi_{n}\right|}
$$

for a set of $2 N+2$ real points $\xi_{n} \in \mathbb{R}$ subject to the ordering $\xi_{1}<\xi_{2}<\cdots<\xi_{2 N+2}$. The parameter $\gamma$ is allowed to take any negative value. The solutions are asymptotic to a single copy of $\mathrm{AdS}_{7} \times S^{4}$, and correspond to M2 branes ending on, or intersecting with, M5 and M5' branes. The holographic duals of these solutions correspond to conformal defects in the 6-dimensional $(2,0)$ theory, and more concretely arise from the insertion of surface operators in that theory.

More concretely, the space-time manifold of the solutions has $2 N+1$ independent noncontractible 4-cycles which are topologically 4-spheres. With the sign convention adopted for $G$ in (8.2), there are $N+1$ cycles supporting M5 brane charges, and $N$ cycles supporting M5' brane charges. On $\Sigma$, the end points of the corresponding curves are the boundary $\partial \Sigma$. A basis may be chosen in terms of curves which join the boundary to the left of $\xi_{n}$ to a point on the boundary to the right of $\xi_{n+1}$. When $n=2 a$ is even we obtain a M5' charge $\mathfrak{M}_{a}^{(2)}$, while when $n=2 b-1$ is odd, we obtain a M5 charge $\mathfrak{M}_{b}^{(3)}$, given by

$$
\begin{array}{ll}
\mathfrak{M}_{a}^{(2)}=\frac{4 \nu_{2} \gamma}{c_{2}^{3}}\left(\xi_{2 a+1}-\xi_{2 a}\right), & a=1, \ldots, N, \\
\mathfrak{M}_{b}^{(3)}=\frac{4 \nu_{3} \gamma}{c_{3}^{3}}\left(\xi_{2 b}-\xi_{2 b-1}\right), & b=1, \ldots, N+1 .
\end{array}
$$

These charges determine a Young tableau, and a corresponding irreducible representation of $\mathrm{SU}(N)$. This Young tableau was conjectured in [17] to describe a surface operator in the corresponding representation of $\mathrm{SU}(N)$, in analogy with the behavior of holographic Wilson lines in 4-dimensional $\mathcal{N}=4$ super Yang-Mills theory [37, 41, 47].

\section{$9 \quad$ Summary and open problems}

We have produced an explicit map between the BPS equations for half-BPS solutions of intersecting M2, M5, and M5' branes in the near-horizon limit, and a 2-dimensional field theory, which we have shown to be integrable, and to possess a Lax pair. We have constructed a large family of solutions to the integrable system and to the supergravity BPS equations, and given their physical interpretation, as far as is presently known. 
However, a full study of the integrable system has not been carried out yet, and is presently the biggest obstacle to a complete classification of all half-BPS solutions of intersection M2, M5, and $\mathrm{M} 5^{\prime}$ branes in the near-horizon limit. We hope that, in this brief article, the mechanics of this integrable system, and the motivation for its further study, have been clearly exposed, so that the purpose of a systematic investigation is now clearly defined.

We conclude by stressing that the analogous problem in 10-dimensional Type IIB on $\mathrm{AdS}_{4} \times$ $S^{2} \times S^{2} \ltimes \Sigma$ or on $\mathrm{AdS}_{2} \times S^{4} \times S^{2} \ltimes \Sigma$ and 6-dimensional Type 4b supergravities on $\mathrm{AdS}_{2} \times$ $S^{2} \ltimes \Sigma$ are, from some points of view, better understood. This is due, in large part, to the fact that the fermion contents of those supergravities is chiral so that the partial differential equations for various reduced metric and flux components become genuine Cauchy-Riemann equations which may be solved in terms of holomorphic or harmonic functions. Indeed, all the supergravities mentioned earlier may be solved in terms of harmonic functions subject to certain constraints $[11,13,15]$.

\section{Acknowledgments}

It is a pleasure to thank Constantin Bachas, John Estes, Michael Gutperle, Drya Krym, and Paul Sorba for fruitful collaborations on the projects reviewed in this paper. I gratefully acknowledge the warm hospitality and the financial support of the Laboratoire de Physique Théorique at the Ecole Normale Supérieure, where part of this work was carried out. Finally, I would like to acknowledge the organizers, Decio Levi, Willard Miller, Yvan Saint-Aubin, and Pavel Winternitz for inviting me to the enjoyable conference and celebration in honor of Luc Vinet at the Centre de Recherches Mathématiques.

\section{References}

[1] Aharony O., Berdichevsky L., Berkooz M., Shamir I., Near-horizon solutions for D3-branes ending on 5branes, Phys. Rev. D 84 (2011), 126003, 19 pages, arXiv:1106.1870.

[2] Aharony O., Bergman O., Jafferis D.L., Maldacena J., $\mathcal{N}=6$ superconformal Chern-Simons-matter theories, M2-branes and their gravity duals, J. High Energy Phys. 2008 (2008), no. 10, 091, 38 pages, arXiv:0806.1218.

[3] Aharony O., Gubser S.S., Maldacena J., Ooguri H., Oz Y., Large $N$ field theories, string theory and gravity, Phys. Rep. 323 (2000), 183-386, hep-th/9905111.

[4] Assel B., Bachas C., Estes J., Gomis J., Holographic duals of $D=3 \mathcal{N}=4$ superconformal field theories, J. High Energy Phys. 2011 (2011), no. 8, 087, 30 pages, arXiv:1106.4253.

[5] Assel B., Bachas C., Estes J., Gomis J., IIB duals of $D=3 \mathcal{N}=4$ circular quivers, J. High Energy Phys. 2012 (2012), no. 12, 044, 47 pages, arXiv:1210.2590.

[6] Bachas C., D’Hoker E., Estes J., Krym D., M-theory solutions invariant under $D(2,1 ; \gamma) \oplus D(2,1 ; \gamma)$, Fortschr. Phys. 62 (2014), 207-254, arXiv:1312.5477.

[7] Bagger J., Lambert N., Modeling multiple M2-branes, Phys. Rev. D 75 (2007), 045020, 7 pages, hep-th/0611108.

[8] Berman D.S., M-theory branes and their interactions, Phys. Rep. 456 (2008), 89-126, arXiv:0710.1707.

[9] Bobev N., Pilch K., Warner N.P., Supersymmetric Janus solutions in four dimensions, J. High Energy Phys. 2014 (2014), no. 6, 058, 49 pages, arXiv:1311.4883.

[10] Boonstra H.J., Peeters B., Skenderis K., Brane intersections, anti-de Sitter space-times and dual superconformal theories, Nuclear Phys. B 533 (1998), 127-162, hep-th/9803231.

[11] Chiodaroli M., D’Hoker E., Guo Y., Gutperle M., Exact half-BPS string-junction solutions in six-dimensional supergravity, J. High Energy Phys. 2011 (2011), no. 12, 086, 58 pages, arXiv:1107.1722.

[12] Cremmer E., Julia B., Scherk J., Supergravity in theory in 11 dimensions, Phys. Lett. B 76 (1978), 409-412.

[13] D'Hoker E., Estes J., Gutperle M., Exact half-BPS type IIB interface solutions. I. Local solution and supersymmetric Janus, J. High Energy Phys. 2007 (2007), no. 6, 021, 60 pages, arXiv:0705.0022. 
[14] D'Hoker E., Estes J., Gutperle M., Exact half-BPS type IIB interface solutions. II. Flux solutions and multi-Janus, J. High Energy Phys. 2007 (2007), no. 6, 022, 56 pages, arXiv:0705.0024.

[15] D'Hoker E., Estes J., Gutperle M., Gravity duals of half-BPS Wilson loops, J. High Energy Phys. 2007 (2007), no. 6, 063, 52 pages, arXiv:0705.1004.

[16] D'Hoker E., Estes J., Gutperle M., Krym D., Exact half-BPS flux solutions in M-theory. I. Local solutions, J. High Energy Phys. 2008 (2008), no. 8, 028, 55 pages, arXiv:0806.0605.

[17] D'Hoker E., Estes J., Gutperle M., Krym D., Exact half-BPS flux solutions in M-theory. II. Global solutions asymptotic to $\mathrm{AdS}_{7} \times S^{4}$, J. High Energy Phys. 2008 (2008), no. 12, 044, 21 pages, arXiv:0810.4647.

[18] D'Hoker E., Estes J., Gutperle M., Krym D., Janus solutions in M-theory, J. High Energy Phys. 2009 (2009), no. 6, 018, 17 pages, arXiv:0904.3313.

[19] D'Hoker E., Estes J., Gutperle M., Krym D., Sorba P., Half-BPS supergravity solutions and superalgebras, J. High Energy Phys. 2008 (2008), no. 12, 047, 72 pages, arXiv:0810.1484.

[20] D'Hoker E., Freedman D.Z., Supersymmetric gauge theories and the AdS/CFT correspondence, hep-th/0201253.

[21] D'Hoker E., Freedman D.Z., Jackiw R., SO(2, 1)-invariant quantization of the Liouville theory, Phys. Rev. D 28 (1983), 2583-2598.

[22] D'Hoker E., Jackiw R., Liouville field theory, Phys. Rev. D 26 (1982), 3517-3542.

[23] D'Hoker E., Vinet L., Supersymmetry of the Pauli equation in the presence of a magnetic monopole, Phys. Lett. B 137 (1984), 72-76.

[24] D'Hoker E., Vinet L., Constants of motion for a spin-1/2 particle in the field of a dyon, Phys. Rev. Lett. 55 (1985), 1043-1046.

[25] D'Hoker E., Vinet L., Spectrum (super-) symmetries of particles in a Coulomb potential, Nuclear Phys. B 260 (1985), 79-102.

[26] Duff M.J. (Editor), The world in eleven dimensions: supergravity, supermembranes and M-theory, Studies in High Energy Physics Cosmology and Gravitation, IOP Publishing, Ltd., Bristol, 1999.

[27] Estes J., Feldman R., Krym D., Exact half-BPS flux solutions in M-theory with $D\left(2,1: c^{\prime}: 0\right)^{2}$ symmetry: local solutions, Phys. Rev. D 87 (2013), 046008, 19 pages, arXiv:1209.1845.

[28] Figueroa-O'Farrill J., Papadopoulos G., Maximally supersymmetric solutions of ten- and eleven-dimensional supergravities, J. High Energy Phys. 2003 (2003), no. 3, 048, 25 pages, hep-th/0211089.

[29] Freedman D.Z., Van Proeyen A., Supergravity, Cambridge University Press, Cambridge, 2012.

[30] Gaiotto D., Witten E., $S$-duality of boundary conditions in $\mathcal{N}=4$ super Yang-Mills theory, Adv. Theor. Math. Phys. 13 (2009), 721-896, arXiv:0807.3720.

[31] Gaiotto D., Witten E., Supersymmetric boundary conditions in $\mathcal{N}=4$ super Yang-Mills theory, J. Stat. Phys. 135 (2009), 789-855, arXiv:0804.2902.

[32] Gaiotto D., Witten E., Janus configurations, Chern-Simons couplings, and the $\theta$-angle in $\mathcal{N}=4$ super Yang-Mills theory, J. High Energy Phys. 2010 (2010), no. 6, 097, 58 pages, arXiv:0804.2907.

[33] Gauntlett J.P., Intersecting branes, in Dualities in Gauge and String Theories (Seoul/Sokcho, 1997), World Sci. Publ., River Edge, NJ, 1998, 146-193, hep-th/9705011.

[34] Gauntlett J.P., Kastor D.A., Traschen J., Overlapping branes in M-theory, Nuclear Phys. B 478 (1996), 544-560, hep-th/9604179.

[35] Gauntlett J.P., Myers R.C., Townsend P.K., Supersymmetry of rotating branes, Phys. Rev. D 59 (1999), 025001, 12 pages, hep-th/9809065.

[36] Gauntlett J.P., Pakis S., The geometry of $D=11$ Killing spinors, J. High Energy Phys. 2003 (2003), no. 4, 039, 33 pages, hep-th/0212008.

[37] Gomis J., Passerini F., Holographic Wilson loops, J. High Energy Phys. 2006 (2006), no. 6, 074, 30 pages, hep-th/0604007.

[38] Gran U., Gutowski J., Papadopoulos G., M-theory backgrounds with 30 Killing spinors are maximally supersymmetric, J. High Energy Phys. 2010 (2010), no. 3, 112, 38 pages, arXiv:1001.1103.

[39] Gustavsson A., Algebraic structures on parallel M2 branes, Nuclear Phys. B 811 (2009), 66-76, arXiv:0709.1260.

[40] Hull C., Holonomy and symmetry in M-theory, hep-th/0305039. 
[41] Okuda T., Trancanelli D., Spectral curves, emergent geometry, and bubbling solutions for Wilson loops, J. High Energy Phys. 2008 (2008), no. 9, 050, 23 pages, arXiv:0806.4191.

[42] Sevrin A., Troost W., Van Proeyen A., Superconformal algebras in two dimensions with $\mathcal{N}=4$, Phys. Lett. B 208 (1988), 447-450.

[43] Smith D.J., Intersecting brane solutions in string and M-theory, Classical Quantum Gravity 20 (2003), R233-R300, hep-th/0210157.

[44] Tseytlin A.A., Harmonic superpositions of M-branes, Nuclear Phys. B 475 (1996), 149-163, hep-th/9604035.

[45] Tseytlin A.A., Composite BPS configurations of p-branes in 10 and 11 dimensions, Classical Quantum Gravity 14 (1997), 2085-2105, hep-th/9702163.

[46] Witten E., String theory dynamics in various dimensions, Nuclear Phys. B 443 (1995), 85-126, hep-th/9503124.

[47] Yamaguchi S., Bubbling geometries for half-BPS Wilson lines, Internat. J. Modern Phys. A 22 (2007), 1353-1374, hep-th/0601089.

[48] Youm D., Localized intersecting BPS branes, hep-th/9902208. 